\title{
Completion of the genome sequence of Rift Valley fever phlebovirus indicates that the $L$ RNA is negative sense or ambisense and codes for a putative transcriptase-replicase
}

Rolf Muller ${ }^{1}$, Claudio Argentini, Michele Bouloy ${ }^{1}$, Christophe Prehaud and David H.L.Bishop* NERC Institute of Virology and Environmental Microbiology, Mansfield Road, Oxford OX1 3SR, UK and ${ }^{1}$ Institut Pasteur, 28 rue du Dr Roux, 75015 Paris, France

Rift Valley fever virus (RVFV) is a member of the Phlebovirus genus of the virus family Bunyaviridae (reviewed in 1). A derivative of the ZH548 isolate of RVFV has been prepared by serial mutagenesis (2). It has attenuating mutations in all three genomic RNA segments (3). Like other phleboviruses and Uukuniemi virus, the 1691 nucleotide small (S) RNA of RVFV MP12 has an ambisense coding strategy in which the viral nucleocapsid protein is made from a subgenomic viralcomplementary mRNA and a second gene product, a nonstructural protein, is made from a subgenomic viral-sense mRNA $(1,4)$. The 3885 nucleotide middle size (M) RNA of RVFV MP12 has a simple negative sense coding arrangement (5). The RVFV M viral-complementary mRNA codes for the precursor to the viral glycoproteins (1). To complete the sequence of the MP12 derivative, the large (L) RNA has been cloned and sequenced. The L RNA is 6606 nucleotides long and has a single long open reading frame coding for 2149 amino acids (243.6 $\mathrm{kDa}$ ) in the viral-complementary sequence. It exhibits some sequence homologies to other viral RNA polymerases. The 5' and $3^{\prime}$ non-coding regions in the viral-complementary RNA sequence are 18 and 141 nucleotides in length, respectively. There is the potential for a short gene product $(3.5 \mathrm{kDa})$ coded in the $5^{\prime}$ region of the viral RNA sequence, i.e., the L RNA, like the RVFV S RNA, may have an ambisense coding strategy. This remains to be confirmed by identification of a gene product. The $3^{\prime}$ and $5^{\prime}$ ends of the L RNA have inverted complementary sequences over a length of 8 nucleotides (5'ACACAAAG..;
..CUUUGUGU $3^{\prime}$ ). Comparison of the L, M, and S RNA sequences indicates that all the RVFV end sequences are conserved (4, 5, Figure 1).

\section{ACKNOWLEDGEMENTS}

R.M. holds a fellowship from the Marcel Merieux foundation; C.A. is a recipient of a fellowship from Cenci Bolognetti, Rome, Italy. The help of B. Caudron is acknowledged.

\section{REFERENCES}

1. Bishop,D.H.L. (1990) In Fields,B.N. et al., (ed.) Virology (2nd edition), Raven Press, New York, pp. 1155-1173.

2. Caplen,H., Peters,C.J. and Bishop,D.H.L. (1985) J. Gen. Virol. 66, $2271-2277$.

3. Salluzzo,J.F. and Smith,J.F. (1990) Vaccine 8, 369-375.

4. Giorgi,C., Accardi,L., Nicoletti,L., Gro,M.C., Takehara,K., Hilditch,C. and Bishop,D.H.L. (1991) Virology 180, 738-753.

5. Takehara,K., Min,M.-K., Battles,J.K., Sugiyama,K., Emery,V.C., Dalrymple,J.M. and Bishop,D.H.L. (1989) Virology 169, 452-457.
L 3' TGTGTTTCCGCGGGTTAGTACCTAGATA...
M 3' TGTGTTTCTGGCCACGTTGAATTTCTCA.....
S 3' TGTGTTTCTGGGGGATCACGAATAGTTC.....
L 5' ACACAAAGGGCCTTATAAATGGAGCCCG.....
M 5' ACACAAAGATGGGCATTAAATGTATGTT.....
S 5' ACACAAAGACCCCCTAGTGATACAAACA.....

Figure 1. Conserved end sequences of RVFV L, M, and S RNAs shown in the DNA form.

* To whom correspondence should be addressed 